\title{
Early and Mid-Term Results of Endovascular Aortic Repair Using a Crossed-Limb Technique for Patients with Severely Splayed Iliac Angulation
}

\author{
Kunihiro Yagihashi, MD, PhD, ${ }^{1}$ Hiroshi Nishimaki, MD, PhD, ${ }^{2}$ Yukihisa Ogawa, MD, PhD, ${ }^{1}$ \\ Kiyoshi Chiba, MD, PhD, ${ }^{2}$ Kenji Murakami, MD, PhD, ${ }^{1}$ Daijun Ro, MD, PhD, ${ }^{2}$ Hirokuni Ono, MD, ${ }^{2}$ \\ Yuka Sakurai, MD, ${ }^{2}$ Takeshi Miyairi, MD, PhD, ${ }^{2}$ and Yasuo Nakajima, MD, PhD1
}

Objective: We evaluated early and mid-term results of endovascular aortic repair (EVAR) using crossed-limb and non-crossed-limb techniques.

Material and Methods: From December 2011 to October 2013, 37 patients (31 men; mean age 75.4 years) were treated with EVAR (crossed-limb, 21 and non-crossed-limb, 16). We compared technical success, maximum short-axis diameter of abdominal aortic aneurysm, iliac angulation, time for catheterization of the short contralateral limb gate of the main body (SCT), and complications between the groups.

Results: The mean follow-up period was $810 \pm 230$ days. The technical success rate was $100 \%$. There was no significant difference between the groups in terms of mean shortaxis diameter. Iliac angulation was significantly wider in the crossed-limb group (53.3 \pm 14.6 vs. 39.4 $\pm 13.0, p=0.0049)$. There was no significant difference between the groups in terms of SCT. Limb occlusion occurred in two cases (one crossed-limb and one non-crossed-limb). There were no aneurysm-related deaths.

Conclusion: There were no differences between the crossed-limb and non-crossed-limb techniques in terms of early and mid-term results of EVAR. A crossed-limb technique can be performed safely without prolonged SCT even in severely splayed iliac angulation cases.

${ }^{1}$ Department of Radiology, St. Marianna University School of Medicine, Kawasaki, Kanagawa, Japan

${ }^{2}$ Department of Cardiovascular Surgery, St. Marianna University School of Medicine, Kawasaki, Kanagawa, Japan

Received: December 14, 2016; Accepted: October 14, 2017 Corresponding author: Kunihiro Yagihashi, MD, PhD. Department of Radiology, St. Marianna University School of Medicine, 2-16-1 Sugao, Miyamae-ku, Kawasaki, Kanagawa 216-8511, Japan

Tel: +81-44-977-8111, Fax: +81-44-977-2931

E-mail: k2yagihashi@marianna-u.ac.jp

(cc) BY-NC-SA (9)2018 The Editorial Committee of Annals of Vascular Diseases. This article is distributed under the terms of the Creative Commons Attribution License, which permits use, distribution, and reproduction in any medium, provided the credit of the original work, a link to the license, and indication of any change are properly given, and the original work is not used for commercial purposes. Remixed or transformed contributions must be distributed under the same license as the original.
Keywords: abdominal aortic aneurysm, stent graft, severe angulation

\section{Introduction}

Endovascular aortic repair (EVAR) is used for majority of abdominal aortic aneurysm (AAA) repairs. ${ }^{1,2)}$ In EVAR, the aortic anatomy influences technical success. For example, severe angulation of the aortic neck, large aneurysmal sac, extremely short proximal neck, and severe angulation of the iliac arteries are all anatomical limitations. Especially in cases with severely splayed iliac arteries, catheterization of the short limb gate via the contralateral access route is difficult, and the graft limb can kink due to the sharp angle between the distal aorta and the common iliac artery.

To overcome this barrier, the crossed-limb technique was developed; in contrast to the usual placement of stent grafts (non-crossed-limb technique) where the iliac limbs extend into the ipsilateral iliac artery, the short limb gate of the stent graft's main body faces the ipsilateral side to facilitate catheterization of the short limb via contralateral access using this technique. Ramaiah et al. ${ }^{3)}$ popularized this crossed-limb technique by demonstrating its utility in cases of severely angulated aortic necks, showing a dramatically reduced acuity of the angle at the aortic neck and the iliac gate. Although this technique has been used for many years, its clinical outcomes, pre-operative computed tomography $(\mathrm{CT})$ configuration, and the time for catheterization of the short contralateral limb gate of the main body (SCT) are not well understood. Therefore, we compared EVAR performed using crossed-limb and noncrossed-limb techniques to evaluate these issues.

\section{Materials and Methods}

This retrospective study was performed at St. Marianna University School of Medicine, Miyamae-ku, Kawasaki, Kanagawa, Japan. The institutional review board ap- 
proved this study and informed consent was waived. From December 2011 to October 2013, 37 patients (31 men; mean age, 75.4 years) were treated with EVAR using an ENDURANT II (Medtronic Vascular, Santa Rosa, CA, USA) stent graft. The technique was chosen on the basis of a pre-operative CT. For example, if the angle between the common iliac arteries (CIAs) was increased and catheterization of the short limb on the contralateral side was expected to be difficult, we chose the crossed-limb technique. However, in other cases our standard non-crossed-limb technique was chosen, in which the contralateral limb gate was positioned anterior to the ipsilateral limb.

Twenty-one patients underwent EVAR with a planned crossed-limb technique, whereas 16 patients underwent the non-crossed-limb technique. All patients were followed after EVAR; the mean follow-up period was $810 \pm 230$ days. Follow-up CT scans were performed at one week, three months, six months, and one year following the procedure, and annually thereafter. We evaluated the following: technical success, maximum short diameter of the AAA in the short-axis plane, iliac angulation on preoperative CT, SCT, graft kinking, type 1 or 3 endoleaks, and death during the follow-up period. Technical success was defined as endograft deployment in the intended position and no angiographic type 1 or 3 endoleaks, limb occlusion, or additional unplanned surgical or endovascular interventions within $24 \mathrm{~h}$ after the EVAR. Iliac angulation was measured as the angle between the CIAs on a 3-D volume rendering image using a CT workstation (Ziosoft,
Inc., Tokyo, Japan).

\section{Statistical analysis}

All statistical analyses were performed using $\mathrm{JMP}^{\circledR}$ v.11 (SAS Institute Inc., Cary, NC, USA) software. Comparisons between the two groups in terms of baseline characteristics for continuous measurements were performed using Student's t-test. Associations between categorical variables were analyzed using the Chi-square test. A p-value $<0.05$ was considered to indicate a significant difference.

\section{Results}

Baseline patient demographics and baseline anatomical characteristics of the study groups are outlined in Table 1. Although there was no significant difference between the groups in terms of the pre-operative mean of the maximum short diameter $(p=0.128)$, there was a significant difference in terms of iliac angulation $(53.3 \pm 14.6$ vs. $39.4 \pm 13.0, p=0.0049)$. All cases were carried out without complications during the procedure, and the technical success was $100 \%$. No type 1 or 3 endoleaks were observed on post-procedure angiographies or on CT scans at one week after EVAR. There was no significant difference between the groups in terms of SCT $(p=0.510)$. The distal fixation sites did not differ significantly between the groups (Table 2). Two iliac limb occlusions occurred during the follow-up period. One limb occlusion occurred at 30 days after EVAR using a crossed-limb technique with

Table 1 Patient demographics data and baseline anatomical characteristics

\begin{tabular}{lccc}
\hline & Crossed-limb group & Non-crossed-limb group & $p$ \\
\hline Age, $y$ & $77.0 \pm 7.2$ & $73.3 \pm 9.0$ & 0.181 \\
Gender, male/female & $17 / 4$ & $14 / 2$ & 0.592 \\
Operation time, minutes & $144.3 \pm 38.5$ & $139.0 \pm 53.1$ & 0.741 \\
Maximum short diameter of AAA, mm & $54.4 \pm 10.0$ & $49.0 \pm 10.8$ & 0.128 \\
Iliac angulation, ${ }^{\circ}$ & $53.3 \pm 14.6$ & $39.4 \pm 13.0$ & 0.0049 \\
Length of CIA, mm $_{\text {Presence of coiling of IIA }}$ & $40.6 \pm 12.9$ & $42.0 \pm 11.5$ & 0.636 \\
\hline
\end{tabular}

Continuous data are presented as means \pm standard deviation; categorical data are given as counts. AAA: abdominal aortic aneurysm; CIA: common iliac artery; IIA: internal iliac artery

Table 2 Endovascular implantation details and follow-up data

\begin{tabular}{lccc}
\hline & Crossed-limb group & Non-crossed-limb group & $p$ \\
\hline SCT, second & $124.8 \pm 26.8$ & $153.4 \pm 33.3$ & 0.510 \\
Distal fixation site & & & 26 \\
CIA & 34 & 6 & 0.863 \\
EIA & 8 & $1(6.25)$ & 0.791 \\
Limb thrombosis & $1(4.76)$ & 0.685 \\
\hline
\end{tabular}

Continuous data are presented as means \pm standard deviation; categorical data are given as counts. SCT: time for catheterization of the short contralateral limb gate of the main body; CIA: common iliac artery; EIA: external iliac artery 
(A)

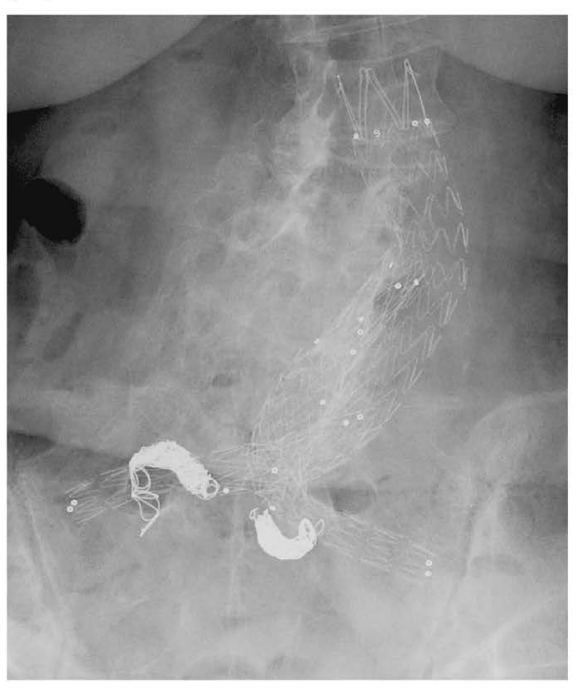

(B)

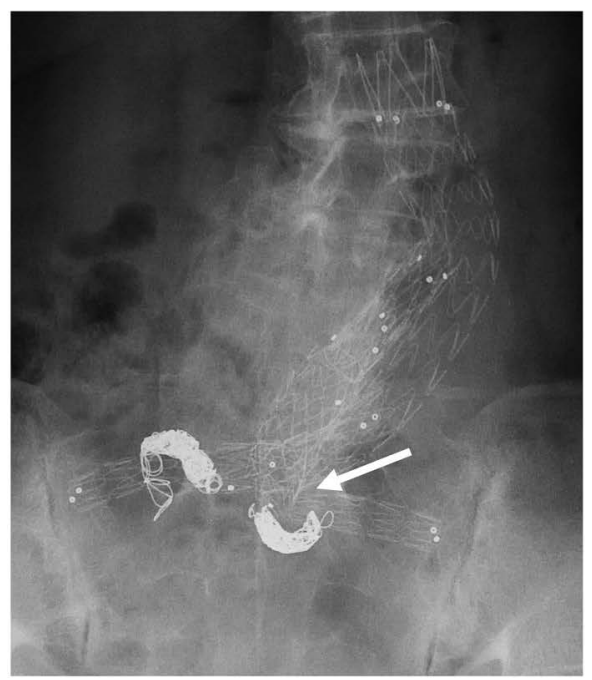

Fig. 1 (A) Post-operative plain abdominal radiograph shows the crossed-limb configuration. (B) Plain abdominal radiograph one month after stent graft placement shows limb kinking (arrow): combination of limb angulation and reduction of the luminal diameter.

(A)

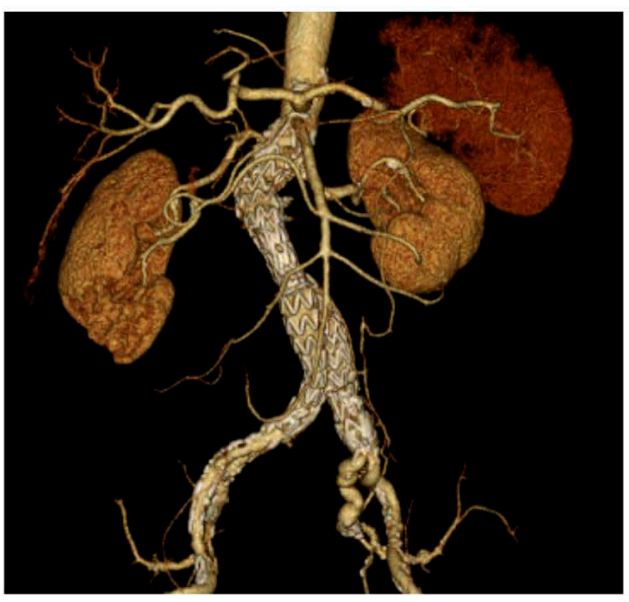

(B)

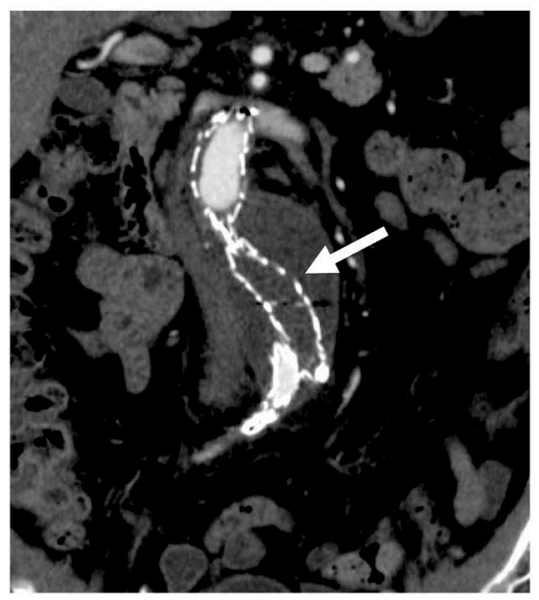

Fig. 2 (A) Three-dimensional computed tomographic (CT) image showing the non-crossedlimb configuration. (B) The CT scan clearly demonstrates the absence of contrast material in the left limb (arrow), indicating limb occlusion of the stent graft.

landing in the external iliac artery (EIA) (case 1, Fig. 1) and the other occurred at 263 days after EVAR using a non-crossed-limb technique with landing in the CIA (case 2, Fig. 2). Both cases were managed with thrombectomy and additional bare stenting of the iliac artery. The remaining endograft limbs were patent without stent fractures or migrations. No perioperative deaths or aneurysm-related deaths occurred in either group.

\section{Discussion}

In this study, there were no differences between the early and mid-term results of EVAR when using the crossed- limb and the non-crossed-limb techniques. SCT in the crossed-limb technique group was not different from that in the non-crossed-limb group, although the iliac angulation was severely and significantly splayed in the crossed-limb group. This suggests that the crossed-limb technique facilitates cannulation of the short limb via the contralateral side in case of severely splayed iliac angulation by decreasing the iliac angulation without prolonging the fluoroscopic time. On the basis of this study, we recommend application of the crossed-limb technique in case of splayed iliac angulation ( $\geq 40$ degrees).

Henretta et al. ${ }^{4}$ and Ramaiah et al. ${ }^{3)}$ reported the crossed-limb technique in 1999 and 2002, respectively, 
and since then it has been employed in patients with severe neck angulation and severely splayed CIAs in order to facilitate the cannulation of the crossed-limb via contralateral access. Consequently, this crossed-limb configuration allows for gentler engagement of the severely splayed CIAs and helps avoid graft kinking. Recently, Georgakarakos et al. ${ }^{5)}$ reported that the hemodynamic behavior of the crossed-limb position resembles that of a typically positioned endograft. This technique also allows a shortened graft length in the aneurysmal sac to avoid covering the internal iliac artery when necessary. Similar to the reports of this study, Georgiadis et al.6) reported no difference between the crossed-limb technique and the conventional endograft position in terms of short- or mid-term clinical outcomes. However, unlike us, they used several kinds of devices (Talent, Medtronic Vascular, Santa Rosa, CA, USA), Endurant (Medtronic Vascular), and Excluder (W. L. Gore \& Associates, Flagstaff, AZ, USA).

We encountered two iliac limb thromboses (one in each group) during the follow-up period. Iliac limb occlusion may be caused by an underlying kinking of the metallic skeleton, extension of the stent graft into the EIA, or migration and dislocation of an endograft limb. ${ }^{7,8)}$ According to previous reports, the presence of small, calcific, or tortuous iliac vessels and EIA landing are also related to graft limb thrombosis. ${ }^{9)}$ To reduce the incidence of graft limb thrombosis, adjunctive bare metal stent insertion has been used ${ }^{10)}$ and was reported to reduce the rate of iliac graft thrombosis from $5 \%$ to $0.9 \% .^{9)}$ Another way to reduce graft thrombosis is to avoid tortuous iliac vessels landing. One of our iliac limb thrombosis cases occurred with an EIA landing in the crossed-limb group (case 1). Pre-operative iliac angulation of this case showed a severely splayed iliac angulation $\left(82.2^{\circ}\right)$ and a tortuous iliac artery. The reason for limb thrombosis in our case was likely a combination of the EIA landing, the severely splayed iliac angulation, and the tortuous iliac artery, despite using a crossed-limb technique. Perhaps it is possible to avoid limb thrombosis by using an additional bare stent for the iliac artery. The other iliac thrombosis occurred in a noncrossed-limb technique with a CIA landing (case 2). However, the iliac angulation was relatively splayed $\left(52.8^{\circ}\right)$. In this case, using the crossed-limb technique might have helped avoid this complication.

There are several limitations to this study. First, we did not evaluate proximal neck angulation or mural thrombi in the aneurysmal sac. These factors would likely affect the cannulation of the contralateral limb via the contralateral site. Second, we used the crossed-limb technique for patients in whom cannulation of the short limb via the contralateral side was expected to be difficult. To evaluate the usefulness of the crossed-limb technique, a prospective study with a control group is needed.

\section{Conclusion}

There were no differences in the early and mid-term results of EVAR when using the crossed-limb and noncrossed-limb techniques. Even with severely splayed iliac angulation, the crossed-limb technique can be performed safely without prolonged SCT. The crossed-limb technique decreases the aortoiliac angulation and, had a low incidence of iliac limb occlusion; however, larger prospective comparative studies are needed to confirm these results.

\section{Acknowledgments}

The authors are grateful to Jay Starkey, MD, St. Luke's International Hospital, for his revision of the English text.

\section{Disclosure Statement}

Hiroshi Nishimaki received travel expenses/gift from Medtronic Japan Co., Ltd., W. L. Gore \& Associates, Co., Ltd., Medico's Hirata Inc., and Japan Lifeline Co., Ltd. Yukihisa Ogawa received honoraria from W. L. Gore \& Associates, Co., Ltd. The other authors have no conflicts of interest.

\section{Author Contributions}

Study conception: HN

Data collection: KY, YO

Analysis: KY, YO

Investigation: $\mathrm{KY}, \mathrm{YO}, \mathrm{KM}, \mathrm{HN}$

Writing: KY

Critical review and revision: all authors

Final approval of the article: all authors

Accountability for all aspects of the work: all authors

\section{References}

1) Dua A, Kuy S, Lee CJ, et al. Epidemiology of aortic aneurysm repair in the United States from 2000 to 2010. J Vasc Surg 2014; 59: 1512-7.

2) Georgakarakos E, Gasser TC, Xenos M, et al. Applying findings of computational studies in vascular clinical practice: fact, fiction, or misunderstanding? J Endovasc Ther 2014; 21: 434-8.

3) Ramaiah VG, Thompson CS, Shafique S, et al. Crossing the limbs: a useful adjunct for successful deployment of the AneuRx stent-graft. J Endovasc Ther 2002; 9: 583-6.

4) Henretta JP, Karch LA, Hodgson KJ, et al. Special iliac artery considerations during aneurysm endografting. Am J Surg 1999; 178: 212-8.

5) Georgakarakos E, Xenakis A, Manopoulos C, et al. Modeling and computational analysis of the hemodynamic effects of crossing the limbs in an aortic endograft ("ballerina” position). J Endovasc Ther 2012; 19: 549-57. 
6) Georgiadis GS, Georgakarakos EI, Antoniou GA, et al. Clinical outcomes after crossed-limb vs. conventional endograft configuration in endovascular AAA repair. J Endovasc Ther 2013; 20: 853-62.

7) van Keulen JW, de Vries JP, Dekker H, et al. One-year multicenter results of 100 abdominal aortic aneurysm patients treated with the Endurant stent graft. J Vasc Surg 2011; 54: 609-15.

8) Maleux G, Koolen M, Heye S, et al. Limb occlusion after endovascular repair of abdominal aortic aneurysms with supported endografts. J Vasc Interv Radiol 2008; 19: 140912.

9) Troisi N, Torsello G, Donas KP, et al. Endurant stent-graft: a 2-year, single-center experience with a new commercially available device for the treatment of abdominal aortic aneurysms. J Endovasc Ther 2010; 17: 439-48.

10) Sivamurthy N, Schneider DB, Reilly LM, et al. Adjunctive primary stenting of Zenith endograft limbs during endovascular abdominal aortic aneurysm repair: implications for limb patency. J Vasc Surg 2006; 43: 662-70. 